\title{
Cognitive Complaints in Patients with Fibromyalgia Versus Older-Aged Women: Case Control Study
}

Jülide Öncü*, Reşat ilişer and Banu Kuran

Department of PM\&R, Şişli Etfal Teaching Hospital, Istanbul, Turkey

\begin{abstract}
Aim of this study: We aimed to show the differences in cognitive complaints and their relations with clinical variables between the subjects with fibromyalgia, age-matched healthy controls and older-aged group with Short Test of Mental Status. By means of covering a broad range of cognitive functions of STMS, mild cognitive impairment in patients with FMS and older-aged women could be demonstrated easily and we could obtain the similar results in other studies using objective neurophsychologic methods without using these tests that was the striking aspect of our study. Considering the difficulty of the objective tests in clinical setting, and since it is important to characterize easily the nature of the dysfunction in clinical setting in order cope with this chronic disorder, our results might be useful in helping patients with FM and older-aged women.
\end{abstract}

Background: this study aimed to compare the complaints of cognitive dysfunction in patients with fibromyalgia(FM) and older-aged women and to detect the relationship of the variables pain, fatigue and sleep problems with these complaints

Methods: 86 patients with FM(between the ages of 25-40), age-matched 75 healthy controls and 80 olderaged women with cognitive complaints(between the ages of 60-75) were analyzed. Perceived level of cognitive impairment was asssesed with the items in Short test of Mental Status(STMS). Measures of depressive symptoms, pain and fatigue severity were obtained from the Beck Depression Inventory(BDI), Visual Analog Scale(VAS) and fatigue severity scale(FSS). And measures of subjective sleep disturbance and relevant demographic data were obtained from an open-ended questionnaire.

Results: Individuals with FM scored significantly lower on total score self-report measures of dyscognition $(28.8+3.3)($ mean+SD) than did healthy controls $(37.6+0.6)($ mean+SD $)(p<0.05)$ and scored similarly in older-aged women $(29.9+3.7)($ mean+SD) $(p>0.05)$. The most severly affected functions were attention and immediate recall in patients with FM however, it was delayed recall in older-aged women. The group of patients with FM showed a lower performance than older-aged women in attention $(p<0.01)$ and immediate recall $(p<0.05)$. However, older-aged group significantly differed from FM group in terms of arithmetic, constructional praxis and abstraction subdimensions of STMS. Fatigue and pain severity were the strongest contributing factors for complaints of cognitive dysfunction in patients with FM but, age and sleep disturbance were the most significant problems influencing the cognitive status in older-aged women $(p<0.05)$.

Conclusion: Mild cognitive impairment was shown to be associated with the two groups studied, but with significant differences between them.

Kewords: Fibromyalgia; Cognitive complaints; Older-aged women

Abbreviations: FMS: Fibromyalgia syndrome; BDI: Beck Depression Inventory; STMS: Short Test of Mental Status; FSS: Fatigue Severity Scale; MCI: Mild Cognitive impairment; VAS:Visual Analog Scale; WPI:Widespread Pain Index; SSS: Symptom Severity Scale; ADL:activity of daily living; ACR: American College of Rheumtaology; SD:Standard Deviation; ANOVA: Analysis of variance

\section{Background}

The primary symptom of fibromiyalgia is chronic, widespread pain accompanied by diffuse tenderness to light palpation however, a decrease in concentration and memory are further complaints that add significantly to the degree of suffering [1]. It is one of the rheumatic illnesses with the greatest impact on patient quality of life, having negative consequences on their capability including intellectual activity, personal relationships and professional career to the extent where the patient requires multiple intervention strategies $[2,3]$.

Patients with FM have reported that they were unable to perform demanding cognitive tasks at their previous level [4]. Especially patients with jobs that have high cognitive/technical demands are likely to perceive their performance in the workplace to be seriously compromised [4]. In addition many of them have stated that cognitive dysfunction was a more disturbing and disabling symptom even than pain [4]. Thus, perceived decreased cognitive ability is an important factor affecting whether some patients can continue to work or return to their daily life with their previous capacity and it should be handled with care during the course of the disease in patients with FM.

Despite the importance of the cognitive symptoms and the experts' recommendation to evaluate them as a part of the standard FM assessment in clinical setting, information on the evaluation of

*Corresponding author: Jülide Öncü, Institution: Department of PM\&R, Şişli Etfal Teaching Hospital, Turkey, Tel: 90507 -231 68 17; E-mail: julideoncu@yahoo.com

Received December 15, 2014; Accepted January 25, 2015; Published February 03, 2015

Citation: Öncü J, ilişer R, Kuran B (2015) Cognitive Complaints in Patients with Fibromyalgia Versus Older-Aged Women: Case Control Study. J Psychiatry 18: 242 doi: $10.4172 / 2378-5756.1000242$

Copyright: (c) 2015 Öncü J, et al. This is an open-access article distributed under the terms of the Creative Commons Attribution License, which permits unrestricted use, distribution, and reproduction in any medium, provided the original author and source are credited 
cognitive status limited to a few studies [5]. These studies mainly used more complicated and less clinically feasible of neuropsychological test evaluations. The number of studies conducted with subjective cognitive tests in patients with chronic pain including FM was quite low $[5,6]$. Andreu at al have evaluated the cognitive complaints by using Mini Mental State Examination which was one of the subjective cognitive tests and found that there was high frequency of cognitive impairment in patients with FM compared with the population reference value [5].

Results from neuropsychological tests showed that patients with FM perform more poorly on a range of cognitive tasks than carefully matched adults of similar age and performed similarly to people 20 years older [7-9]. But, up to date, there is no study exist in the literature comparing the subjective complaints of FM patients with older-aged and healthy age-matched groups. So; the objectives of this analysis were as follows: First, to evaluate the severity and distribution of cognitive complaints in patients with FM and older-aged group using a brief and quantitative subjective measure of the cognitive status in adults such as the Short Test of Mental Status. Second; to determine whether some differences exist between these groups in terms of cognitive subdimensions and their relations with clinical and demographical variables.

\section{Methods}

After the study was reviewed and approved by a Clinical Research Ethics Committee and conducted in compliance with the Declaration of Helsinki; an informed consent, compliant with the Sisli Etfal Teaching Hospital Ethics Board requirements was obtained from all participants prior to the study inclusion.

We recruited 96 women between the ages of 20 and 45 from our outpatient clinic who were diagnosed as fibromyalgia syndrome according to ACR 2010 and ACR 1990 diagnostic criteria. Subjects were accepted as fibromyalgia according to ACR 2010 preliminary diagnostic criteria, if the following 3 conditions were met: 1) Widespread pain index $>7$ and symptom severity scale score $>5$ or WPI between 3 and 6 and SS scale score $>9$. 2) Symptoms have been present at a similar level for at least 3 months. 3) The patient does not have a disorder that would otherwise explain pain [10]. Physicians were also asked to perform tender point count between 0 and 18, described by ACR in 1990 [11]. A pain-free healthy 75 subjects (control group) matched for age and sex and 93 subjects with the age of 60 and 75 years (older-aged women group) were included. Medical examination of the patients included detailed rheumatologic, neurologic and psychiatric examinations performed by specialists of these fields and laboratory analysis including a complete blood cell count, erhytrocyte sedimentation rate, rheumatoid factor, parathyroid hormone, thyroid hormone, cyanocobalamine and folate levels and a blood chemistry panel were also performed. According to the results of these examinations, patients with severe neurologic diseases (such as Alzheimer disease, Parkinson disease, dementia and etc..) and psychiatric disorders (such as depression, bipolar disease, psychosis, neurosis and etc...) having a need of treatment (10 subjects in FM group and 7 subjects in older-aged women) were excluded from the study.

Older-aged women $(n=86)$ complaining of cognitive dysfunction without restricting normal activity of daily living were selected. Observed cognitive impairment was considered present if the participant's friend or family member reported at least one of the following problems during the preceding six months: "difficulty remembering things that happened recently, in the past few hours or days;" "forgotten conversations that occurred a few hours or days earlier;" "asked the same questions repeatedly;" "forgotten to turn off the stove;" and "repeated herself more". Participants and proxies were independently asked nine Yes/No questions about ADL deficits (bathing, grooming, feeding, dressing, preparing meals, simple household tasks, operating simple household appliances, shopping, handling money) occurring over the preceding six-month period related to their cognitive functioning. We considered ADL impairment absent if both participant and her proxy agreed that these nine functions were not impaired [12].

The Short Test of Mental Status (STMS) was used to evaluate the subjective cognitive impairments of the patient groups in our study. It was specifically developed for use in dementia assessment and was intended to be more sensitive to problems of learning and mental agility that may be seen in mild cognitive impairment (MCI) [13]. The Short Test of Mental Status (STMS) tests an individual's orientation, attention, immediate recall, arithmetic, abstraction, construction, information, and delayed (approximately 3 minutes) recall [13]. Scores in the 34 to 38 range are essentially normal; scores from 29 to 33 suggestive of MCI, and below 29 , suggestive of dementia. While this test was initially developed to distinguish dementia from normal cognitive function, it has been found to be also helpful in the evaluation of MCI $[13,14]$.

The association of the cognitive complaints with the following variables were also assessed: age, educational level, marrital status, sleep problems, fatigue and pain severity, depressive symptoms and disease duration. To assess pain intensity, subjects were asked to rate their average pain due to $\mathrm{FM}$ over the past 24 hours with an $10 \mathrm{~cm}$ visual analog scale ranging from $0 \mathrm{~cm}$ (indicating no pain) to $10 \mathrm{~cm}$ (indicating pain as bad as you can imagine). Higher scores indicate greater pain severity [15]. For a global assessment of fatigue severity, we used a validated Turkish version of Fatigue severity scale in patients with FM(FSS) [16]. It consists of 9 statements that are scored on a 7-point Likert scale, ranging from 1 (strongly disagree) to 7 (strongly agree) a total score is calculated as the mean score of the 9 statements [16]. A lower total score indicates less effect of fatigue on everyday life [16]. Depressive symptoms in all of the groups were obtained from the Beck Depression Inventory (BDI) [17]. It consists of 21 groups of items that assess both cognitive/affective and vegetative signs of depression. Sleep disturbance was measured using a 100 -mm VAS with the question 'How would you rate the average amount of sleep you have had over the last seven days?'. This VAS had no calibration marks and end descriptors 'Full nights sleep' and 'No sleep at all'. A similar method has been used previously in studies examining the patients with Rheumatoid Arthritis and Osteoarthritis [18].

\section{Statistical Analysis}

Means, standard deviations (SD), medians, and ranges were calculated for continuous variables and frequency counts and percentages were calculated for categorical variables. To evaluate the correlation between these variables pearson correlation analysis was performed. Relationship between variables was evaluated with ANOVA variance analysis. All analyses were performed using SPSS version 16.0 and statistical significance was evaluated at the 0.05 level.

\section{Results}

All patients were female; similar in educational level and marrital status. The mean age of the patients with FM was lower than that of older-aged women $(p<0.05)$ but it was similar to control group $(p>0.05)$. Symptom Severity Score was $9.5+1.5($ mean $+\mathrm{SD})$; Widespread Pain Index score was $11.7+3.1($ mean $+\mathrm{SD})$; the count of tender points was 
Citation: Öncü J, ilişer R, Kuran B (2015) Cognitive Complaints in Patients with Fibromyalgia Versus Older-Aged Women: Case Control Study. J Psychiatry 18: 242 doi: 10.4172/2378-5756.1000242

Page 3 of 6

9.6+3.3 (mean+SD); the disease duration was $1.1+0.6$ years $($ mean $+\mathrm{SD})$ in FM group. Differences between the groups with regarding the clinical and demographic characteristics were shown in Table 1.

Individuals with FM scored significantly lower on total score self-report measures of dyscognition $(28.8+3.3)($ mean+SD) than did healthy controls $(37.6+0.6)($ mean $+\mathrm{SD})(\mathrm{p}<0.05)$. However it was similar to older-aged women $(29.9+3.7)($ mean $+S D)(p>0.05)$. The means and SD's for the items assessing cognitive complaints involved in each section were as shown in (Table 1). Overall $69 \%$ of the subjects with FM and $71 \%$ of the older-aged women complained of mild; $5 \%$ of the patients with FM and $6 \%$ of the older-aged women reported severe cognitive problems. The most commonly noted problem was "trouble in attention (78.2\%) and immediate recall" (66.5\%) for patients with
FM and "delayed recall" (70.1\%) for older-aged women. The one-way ANOVA test showed significant differences between the groups of our patients. The group of patients with FM showed a lower performance as compared with the control group in all of the subsets of STMS except abstraction, information, construction and orientation (Table $1)$. And they showed a lower performance when compared with the older-aged women in attention $(\mathrm{p}<0.01)$ and immediate recall $(\mathrm{p}<0.05)$ (Table 1). The group of patients with older-aged women showed a lower performance when compared with the control group in all of the subtests except orientation, information, attention $(\mathrm{p}<0.05)($ Table 1$)$.

Pearson correlation analysis used to identify unique relationships between types of dyscognition and the clinical presentation of FM (Table 2) and older-aged women (Table 3). A significant relationship

\begin{tabular}{|c|c|c|c|c|}
\hline Characteristics & FMS $(n=86)$ & Older-aged Women $(n=80)$ & Control (n=75) & $P$ value \\
\hline Age (years) & $32.3 \pm 6.0$ & $67.8 \pm 7.2$ & $32.1 \pm 7.7$ & $.037^{\mathrm{bc}}$ \\
\hline Education (years) & $10.1 \pm 2.7$ & $10.6 \pm 3.1$ & $10.5 \pm 2.0$ & .087 \\
\hline Marrital status ( $\%$ married) & 88 & 89 & 85 & .085 \\
\hline VAS-pain & $8.9 \pm 2.2$ & $5.2 \pm 2.0$ & $1.2 \pm 0.1$ & $.035^{\mathrm{abc}}$ \\
\hline VAS-sleep problems & $5.5 \pm 1.1$ & $7.9 \pm 2.5$ & $1.4 \pm 0.3$ & $.041^{\mathrm{abc}}$ \\
\hline FSS & $8.8 \pm 2.1$ & $4.7 \pm 1.5$ & $1.0 \pm 0.0$ & $.039^{a b c}$ \\
\hline BDI & $13.3 \pm 6.0$ & $14.2 \pm 7.1$ & $6.1 \pm 2.1$ & $.021^{\mathrm{ab}}$ \\
\hline STMS total & $28.8 \pm 3.3$ & $28.0 \pm 3.7$ & $37.6 \pm 0.6$ & $.029^{\mathrm{ab}}$ \\
\hline STMS orientation & $4.1 \pm 0.8$ & $4.3 \pm 0.6$ & $4.3 \pm 0.8$ & .066 \\
\hline STMS attention & $2.4 \pm 0.5$ & $5.1 \pm 0.7$ & $5.2 \pm 0.5$ & $.010^{\mathrm{ac}}$ \\
\hline STMS immediate recall & $1.5 \pm 0.3$ & $3.6 \pm 0.8$ & $5.3 \pm 0.3$ & $\begin{array}{l}.002^{\mathrm{ab}} \\
.035^{\mathrm{c}}\end{array}$ \\
\hline STMS delayed recall & $2.3 \pm 0.9$ & $2.4 \pm 0.8$ & $5.2 \pm 0.9$ & $.007^{\mathrm{ab}}$ \\
\hline STMS arithmetic & $3.9 \pm 1.3$ & $2.5 \pm 1.2$ & $5.0 \pm 1.3$ & $.040^{\mathrm{abc}}$ \\
\hline STMS abstraction & $4.8 \pm 0.5$ & $2.5 \pm 0.6$ & $5.3 \pm 0.6$ & $.045^{\mathrm{bc}}$ \\
\hline STMS construction & $4.9 \pm 1.0$ & $2.6 \pm 1.1$ & $5.0 \pm 1.0$ & $.036^{\mathrm{bc}}$ \\
\hline STMS information & $4.5 \pm 1.0$ & $4.9 \pm 1.2$ & $5.1 \pm 1.0$ & .054 \\
\hline
\end{tabular}

FMS: fibromyalgia syndrome; VAS: visual analog scale; FSS: fatigue severity scale; BDI: Beck depression inventory; STMS: short test of mental status. All data presented are mean \pm SD except marrital status which was presented as percentage $(\%)$

asignificant difference between FMS and control groups.

'Significant difference between Older-aged women and control groups.

'Significant difference between FMS and Older-aged women groups (one-way ANOVA and post hoc test. Kruskal Wallis ANOVA and Mann Whitney U test). Table 1: The comparison of demographic and clinical parameters between the groups.

\begin{tabular}{|c|c|c|c|c|c|c|c|c|c|c|c|}
\hline & Age & education & $\begin{array}{l}\text { Marrital } \\
\text { status }\end{array}$ & $\begin{array}{l}\text { Disesae } \\
\text { duration }\end{array}$ & sss & WPI & FM & VAS-pain & BDI & FSS & $\begin{array}{l}\text { VAS- } \\
\text { sleep }\end{array}$ \\
\hline $\begin{array}{l}\text { STMS total-r value } \\
\text { P value }\end{array}$ & $\begin{array}{l}-.074 \\
.599\end{array}$ & $\begin{array}{l}.118 \\
.463\end{array}$ & .032 & $\begin{array}{l}.138 \\
.325\end{array}$ & $\begin{array}{l}-.347 \\
.037^{*}\end{array}$ & $\begin{array}{l}-.030 \\
.830\end{array}$ & $\begin{array}{l}.079 \\
.573\end{array}$ & $\begin{array}{l}-.369 \\
.008^{* *}\end{array}$ & $\begin{array}{l}-.086 \\
.542\end{array}$ & $\begin{array}{l}-.360 \\
.009^{* *}\end{array}$ & $\begin{array}{l}-.341 \\
.049^{*}\end{array}$ \\
\hline $\begin{array}{l}\text { STMS orientation-r value } \\
\text { P value }\end{array}$ & $\begin{array}{l}-.134 \\
.338\end{array}$ & $\begin{array}{l}.105 \\
.455\end{array}$ & $\begin{array}{l}.046 \\
.743\end{array}$ & $\begin{array}{l}-.019 \\
.890\end{array}$ & $\begin{array}{l}-.124 \\
.075\end{array}$ & .021 & $\begin{array}{l}.153 \\
.274\end{array}$ & $\begin{array}{l}.145 \\
.299\end{array}$ & $\begin{array}{l}.008 \\
.954\end{array}$ & $\begin{array}{l}-.125 \\
.371\end{array}$ & $\begin{array}{l}-.183 \\
.190\end{array}$ \\
\hline $\begin{array}{l}\text { STMS attention- } r \text { value } \\
P \text { value }\end{array}$ & $\begin{array}{l}.040 \\
.775\end{array}$ & $\begin{array}{l}.001 \\
.996\end{array}$ & $\begin{array}{l}.192 \\
.169\end{array}$ & $\begin{array}{l}.002 \\
.991\end{array}$ & $\begin{array}{l}-.300 \\
.032^{*}\end{array}$ & $\begin{array}{l}.036 \\
.800\end{array}$ & $\begin{array}{l}.147 \\
.295\end{array}$ & $\begin{array}{l}-.391 \\
.004^{* *}\end{array}$ & $\begin{array}{l}.071 \\
.616\end{array}$ & $\begin{array}{l}-.402 \\
.001^{* *}\end{array}$ & $\begin{array}{l}-.336 \\
.043^{*}\end{array}$ \\
\hline STMS immediate recall $-r$ value $P$ value & $\begin{array}{l}.153 \\
.274\end{array}$ & $\begin{array}{l}-.116 \\
.408\end{array}$ & $\begin{array}{l}.133 \\
.343\end{array}$ & $\begin{array}{l}.187 \\
.180\end{array}$ & $\begin{array}{l}-.312 \\
.033^{*}\end{array}$ & $\begin{array}{l}.093 \\
.509\end{array}$ & .222 & $\begin{array}{l}-.407 \\
.002\end{array}$ & $\begin{array}{l}.109 \\
.437\end{array}$ & $\begin{array}{l}-.374 \\
.006 *\end{array}$ & $\begin{array}{l}-.322 \\
.047^{*}\end{array}$ \\
\hline $\begin{array}{l}\text { STMS delayed recall- } r \text { value } \\
\text { P value }\end{array}$ & $\begin{array}{l}.145 \\
.299\end{array}$ & $\begin{array}{l}-.169 \\
.226\end{array}$ & $\begin{array}{l}.185 \\
.186\end{array}$ & .062 & $\begin{array}{l}.320 \\
.034^{*}\end{array}$ & $\begin{array}{l}.181 \\
.196\end{array}$ & $\begin{array}{l}.227 \\
.102\end{array}$ & $\begin{array}{l}-.387 \\
.003^{* *}\end{array}$ & $\begin{array}{l}-.063 \\
.652\end{array}$ & $\begin{array}{l}-.383 \\
.005^{* *}\end{array}$ & $\begin{array}{l}-.315 \\
.046^{*}\end{array}$ \\
\hline $\begin{array}{l}\text { STMS arithmetic-r value } \\
\text { P value }\end{array}$ & $\begin{array}{l}.008 \\
.957\end{array}$ & $\begin{array}{l}-.096 \\
.495\end{array}$ & $\begin{array}{l}.245 \\
.077\end{array}$ & $\begin{array}{l}.231 \\
.096\end{array}$ & .147 & $\begin{array}{l}.253 \\
.068\end{array}$ & $\begin{array}{l}.156 \\
.264\end{array}$ & 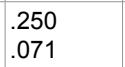 & $\begin{array}{l}.152 \\
.276\end{array}$ & $\begin{array}{l}.147 \\
.293\end{array}$ & $\begin{array}{l}.193 \\
.167\end{array}$ \\
\hline $\begin{array}{l}\text { STMS abstraction- } r \text { value } \\
\text { P value }\end{array}$ & $\begin{array}{l}-.019 \\
.890\end{array}$ & $\begin{array}{l}-.255 \\
.065\end{array}$ & $\begin{array}{l}.216 \\
.121\end{array}$ & $\begin{array}{l}.007 \\
.960\end{array}$ & .040 & $\begin{array}{l}.183 \\
.189\end{array}$ & $\begin{array}{l}-.134 \\
.337\end{array}$ & $\begin{array}{l}-.086 \\
.542\end{array}$ & $\begin{array}{l}.096 \\
.493\end{array}$ & $\begin{array}{l}.183 \\
.189\end{array}$ & .226 \\
\hline $\begin{array}{l}\text { STMS construction-r value } \\
\text { P value }\end{array}$ & $\begin{array}{l}.113 \\
.214\end{array}$ & $\begin{array}{l}-.123 \\
.420\end{array}$ & $\begin{array}{l}.143 \\
.343\end{array}$ & $\begin{array}{l}.181 \\
.183\end{array}$ & $\begin{array}{l}.020 \\
.990\end{array}$ & $\begin{array}{l}.087 \\
.578\end{array}$ & $\begin{array}{l}.142 \\
.270\end{array}$ & $\begin{array}{l}-.127 \\
.428\end{array}$ & $\begin{array}{l}.132 \\
.340\end{array}$ & $\begin{array}{l}.032 \\
.901\end{array}$ & $\begin{array}{l}.043 \\
.529\end{array}$ \\
\hline $\begin{array}{l}\text { STMS information-r value } \\
\text { P value }\end{array}$ & $\begin{array}{l}.125 \\
.210\end{array}$ & $\begin{array}{l}-.171 \\
.228\end{array}$ & $\begin{array}{l}.180 \\
.179\end{array}$ & $\begin{array}{l}.062 \\
.661\end{array}$ & $\begin{array}{l}.231 \\
.150\end{array}$ & $\begin{array}{l}.120 \\
.196\end{array}$ & $\begin{array}{l}.109 \\
.290\end{array}$ & $\begin{array}{l}-.170 \\
.220\end{array}$ & $\begin{array}{l}.125 \\
.126\end{array}$ & $\begin{array}{l}.220 \\
.172\end{array}$ & $\begin{array}{l}.191 \\
.156\end{array}$ \\
\hline
\end{tabular}

FMS: fibromyalgia syndrome; VAS: visual analog scale; FSS: fatigue severity scale; BDI: Beck depression inventory; STMS: short test of mental status; TP: fibromyalgia tender points; SSS:symptom severity score; WPI:widespread pain index; FIQ:Fibromyalgia impact questionairre. ${ }^{*} \mathrm{p}<0.05 ;{ }^{* *} \mathrm{p}<0.01$

Table 2: The results of correlation analysis between subtypes of dyscognition and clinical parameters in patients with fibromyalgia ( $n=86)$ 


\begin{tabular}{|c|c|c|c|c|c|c|c|}
\hline & Age & Education & Marrital status & VAS-pain & BDI score & FSS score & VAS-sleep \\
\hline $\begin{array}{l}\text { STMS total-r value } \\
\text { P value }\end{array}$ & $\begin{array}{l}-.392 \\
.031^{*}\end{array}$ & $\begin{array}{l}.128 \\
.435\end{array}$ & $\begin{array}{l}.065 \\
.675\end{array}$ & $\begin{array}{l}-.275 \\
.048^{\star}\end{array}$ & $\begin{array}{l}.170 \\
.289\end{array}$ & $\begin{array}{c}-.209 \\
.060\end{array}$ & $\begin{array}{l}-.452 \\
.041^{*}\end{array}$ \\
\hline $\begin{array}{l}\text { STMS orientation-r value } \\
\text { P value }\end{array}$ & $\begin{array}{l}-.125 \\
.230\end{array}$ & $\begin{array}{l}.200 \\
.351\end{array}$ & $\begin{array}{l}.045 \\
.731\end{array}$ & $\begin{array}{l}.155 \\
.308\end{array}$ & $\begin{array}{l}.015 \\
.935\end{array}$ & $\begin{array}{c}-.159 \\
.319\end{array}$ & $\begin{array}{l}-.192 \\
.186\end{array}$ \\
\hline $\begin{array}{l}\text { STMS attention- } r \text { value } \\
P \text { value }\end{array}$ & $\begin{array}{r}-.055 \\
.788\end{array}$ & $\begin{array}{l}.206 \\
.101\end{array}$ & $\begin{array}{l}.227 \\
.102\end{array}$ & $\begin{array}{l}-.159 \\
.319\end{array}$ & $\begin{array}{l}.148 \\
.355\end{array}$ & $\begin{array}{r}-.162 \\
.283\end{array}$ & $\begin{array}{l}.085 \\
.836\end{array}$ \\
\hline $\begin{array}{l}\text { STMS immediate recall }-r \text { value } \\
\text { P value }\end{array}$ & $\begin{array}{l}-.359 \\
.036^{*}\end{array}$ & $\begin{array}{l}.153 \\
.353\end{array}$ & $\begin{array}{l}.156 \\
.284\end{array}$ & $\begin{array}{l}.159 \\
.205\end{array}$ & $\begin{array}{c}-.012 \\
.809\end{array}$ & $\begin{array}{l}.185 \\
.309\end{array}$ & $\begin{array}{l}-.258 \\
.048^{*}\end{array}$ \\
\hline $\begin{array}{l}\text { STMS delayed recall- } r \text { value } \\
\text { P value }\end{array}$ & $\begin{array}{l}-.348 \\
.039^{*}\end{array}$ & $\begin{array}{l}.188 \\
.189\end{array}$ & $\begin{array}{l}-.145 \\
.335\end{array}$ & $\begin{array}{l}.211 \\
.048^{*}\end{array}$ & $\begin{array}{l}.082 \\
.801\end{array}$ & $\begin{array}{l}.229 \\
.077\end{array}$ & $\begin{array}{l}.352 \\
.035^{*}\end{array}$ \\
\hline $\begin{array}{l}\text { STMS arithmetic-r value } \\
\text { P value }\end{array}$ & $\begin{array}{l}-.305 \\
.023^{*}\end{array}$ & $\begin{array}{l}.250 \\
.398\end{array}$ & $\begin{array}{l}.128 \\
.280\end{array}$ & $\begin{array}{l}-.381 \\
.046^{*}\end{array}$ & $\begin{array}{l}.196 \\
.380\end{array}$ & $\begin{array}{l}.250 \\
.398\end{array}$ & $\begin{array}{l}-.298 \\
.039^{*}\end{array}$ \\
\hline $\begin{array}{l}\text { STMS abstraction- } r \text { value } \\
P \text { value }\end{array}$ & $\begin{array}{l}-.278 \\
.030^{*}\end{array}$ & $\begin{array}{l}.250 \\
.355\end{array}$ & $\begin{array}{l}.087 \\
.608\end{array}$ & $\begin{array}{l}-.305 \\
.049^{*}\end{array}$ & $\begin{array}{l}.062 \\
.650\end{array}$ & $\begin{array}{r}-.212 \\
.069\end{array}$ & $\begin{array}{l}-.325 \\
.040^{*}\end{array}$ \\
\hline $\begin{array}{l}\text { STMS construction- } r \text { value } \\
P \text { value }\end{array}$ & $\begin{array}{l}-.125 \\
.230\end{array}$ & $\begin{array}{l}.100 \\
.951\end{array}$ & $\begin{array}{l}.055 \\
.629\end{array}$ & $\begin{array}{l}.213 \\
.093\end{array}$ & $\begin{array}{l}.040 \\
.875\end{array}$ & $\begin{array}{r}-.022 \\
.709\end{array}$ & $\begin{array}{r}-.019 \\
.811\end{array}$ \\
\hline $\begin{array}{l}\text { STMS information-r value } \\
P \text { value }\end{array}$ & $\begin{array}{r}-.134 \\
.265\end{array}$ & $\begin{array}{l}.200 \\
.351\end{array}$ & $\begin{array}{l}.064 \\
.631\end{array}$ & $\begin{array}{l}-.160 \\
.120\end{array}$ & $\begin{array}{l}.015 \\
.935\end{array}$ & $\begin{array}{l}.221 \\
.086\end{array}$ & $\begin{array}{r}-.035 \\
.608\end{array}$ \\
\hline
\end{tabular}

BDI: Beck Depression Inventory; VAS:Visual Analog Scale; FSS:Fatigue Severity Scale; STMS: Short Test of Mental Status;TPC: Tender Point Count.; ${ }^{\star}$ < 0.05 Table 3: The results of correlation analysis between subtypes of dyscognition and clinical parameters in older-aged women ( $n=80)$.

\begin{tabular}{|c|c|c|c|c|c|c|c|c|}
\hline & Age & VAS-pain & $\begin{array}{l}\text { VAS-sleep } \\
\text { problems }\end{array}$ & FSS & BDI & sss & WPI & TPC \\
\hline STMS total-FM & $.982 p=0.651$ & $\begin{array}{c}.251^{*} \\
p=0.022\end{array}$ & $.123 p=0.081$ & $.190^{*} p=0.035$ & $.977 p=0.634$ & $\begin{array}{c}.164^{*} \\
p=0.041\end{array}$ & $.771 p=0.576$ & $.491 p=0.485$ \\
\hline $\begin{array}{l}\text { STMS total-older } \\
\text { aged women }\end{array}$ & $.263^{*} p=0.025$ & $.405 p=0.415$ & $\begin{array}{c}.196^{*} \\
\mathrm{p}=0.032\end{array}$ & $.436 p=0.472$ & $.902 p=0.602$ & & & \\
\hline
\end{tabular}

BDI: Beck Depression Inventory; VAS:visual Analog Scale; FSS:Fatigue Severity Scale; STMS: Short Test of Mental Status;TPC: Tender Point Count.; "p<0.05.

Table 4: The results of regression analysis in FM and older-aged women groups.

was found between total cognitive score and VAS-pain( $\mathrm{p}<0.01)$, VASsleep $(\mathrm{p}<0.05), F S S(\mathrm{p}<0.01)$, SSS $(\mathrm{p}<0.05)$, while WPI and tender point count were not found to be significantly related with cognitive scores. Correlational analysis also indicated that in older-aged women group; pain intensity, age, sleep and fatigue were all positively related to cognitive complaints (with total score of STMS) $(\mathrm{p}<0.05)$ (Table 3). There weren't any significant relationship between Beck Depression total score and all parameters $(\mathrm{p}>0.05)$.

Simultaneous regression analysis showed that all factors combined accounted for $52 \%$ of the variance in FM group and $48 \%$ in older-aged women in self-report of cognitive difficulties (Table 4). Inspection of the independent contribution of each variable to the prediction of cognitive complaints in older-aged group, controlling for the other variables in the equation, indicated that 2 contributed significantly to the report of cognitive complaints (total score). Age made the greatest contribution $(\mathrm{r}=.26, \mathrm{P}<.05)$, according to the standardized regression coefficient. Self-report of sleep problems made the next highest contribution $(\mathrm{r}=.19, \mathrm{P}<.05)$. In FM group, FSS, pain severity according to VAS and symptom severity score had a significant unique contribution to the report of cognitive complaints in the following order of importance: pain severity $(\mathrm{r}=.25, \mathrm{P}<.05)$, fatigue $(\mathrm{r}=.19, \mathrm{P}<.05)$, symptom severity score $(\mathrm{r}=.16, \mathrm{P}<.05)$. Further, the sleep problems $(\mathrm{r}=.12, \mathrm{P}<.08)$ contributed only marginally to the report of the cognitive complaints.

\section{Discussion}

These results confirm the existence of mild cognitive impairment in both groups (FM and older-aged women) regarding the cutoff points of the cognitive performance normality of Short Test of Mental Status (STMS). Although the level of perceived cognitive dysfunction were found similar in both patient groups, with some significant differences between them. In patients with FM attention and immediate memory were among the most severe cognitive complaints; however, abstraction, construction, arithmetic and delayed memory were the subsets most significantly impaired in older-aged group. In general, fatigue and pain severity were the strongest contributing factors for complaints of cognitive function in patients with FM but, age and sleep disturbance were the most significant problems influencing the cognitive status in older-aged women.

Although we examined self-reported cognitive complaints among patients with FM and older-aged women and the study lacked an objective neuropsychologic assessment; our results showed that there was demonstrable cognitive dysfunction with STMS in both groups. STMS was developed and validated as a screening bedside mental status test and despite the lack of validity data, it has been used to identify subjective cognitive dysfunction among patients with mental disorders, and the items do show adequate internal consistency [19]. Supporting these findings, different cognitive tests have been used to evaluate subjective cognitive dysfunction in chronic pain patients including fibromyalgia $[5,6]$. Roth et al used Brief Symptom Inventory for the assessment of cognitive complaints in patients with chronic pain and reported that although it was the measure of the severity of psychological impairment its' cognitive items identified the cognitive problems in patients with chronic pain [6]. Andreu et al assessed memory function in patients with fibromyalgia with Mini Mental State Examination (MMSE) and they noted that fibromyalgia patients have reported more severe cognitive impairment when compared with other patients with chronic pain and healthy controls [5]. In a study of particular relevance to the present discussion, authors have suggested to use STMS rather than MMSE [20]. In this study comparing these two screening tests, they concluded that although MMSE was the most widely used one, STMS was found to be more sensitive to detect mild cognitive impairment than MMSE [20]. Taken these results and 
also the difficulty of objective testing methods in clinical practice into consideration, STMS test might be useful screening test to distinguish mild cognitive impairment in subjects with FM and older-aged women from healthy controls.

In our study only $5 \%$ of the patients with FM and $6 \%$ of the older aged women reported severe cognitive impairment and majority of neither FM subjects nor older-aged women were found as demented. Their mean scores of STMS obtained as 28.8 for FM and 28.0 for older aged women however, such performances were below the expected range in the study pointing out mild cognitive impairment. These data are comparable to those reported by Pericot et al who have already showed the evidence of mild cognitive impiarment in 29 of 32 women (90.5\%) with FM and this subjective complaint of memory loss was also confirmed by neuropsychological evaluation [21]. Also, regarding the similarity of total cognitive scores of FM and older aged women, our findings did not differ from the studies in the literature; since there is a growing body of evidence indicating that patients with FM had poorer cognitive performance than carefully matched adults of similar age and performed similarly to people 20 years older [22-24].

Although our study seemed to replicate the results of the studies that were previously mentioned; it has been shown that some differences were present in the distributions of these complaints between the groups. Patients with FM had lower performance in subsets of attention and memory-related complaints. This evidence is consistent with the findings in the literature and in many studies this pattern of the results have been shown to be consistent with impairment in a particular kind of cognitive ability called executive control that resulted into disability in particularly managing multiple tasks and switching attention quickly $[25,26]$. On the other hand; in older-aged group it has been shown that particularly slower information processing speed was critical in many theories of cognitive aging that might be a different mechanism from FM [27]. In our study fund of abstraction, arithmetic, delayed recall and constructional praxis score showing information processing speed were found to be lower in older-aged group than that of FM like others have supported $[27,28]$. Using data from elderly with no disease living in the community, there is evidence that cognitive speed and memory performance decline with age, but that crystallized abilities remain largely intact in those who survive for long-term follow-up [29]. However, this was not the case for FM, because they performed just as well as the age-matched controls and were significantly faster than the older-aged group in our study. This might suggest that subjects with FM have specific neurologic/cognitive deficits, rather than global neurologic decline that is more characteristic of older adults. It also makes us optimistic that the deficits may be remedial or temporary, although this has not been supported by any data yet.

And also our study included some disease-related variables having reasonablelikelihood of sharing significant relation with these complaints which were not contained in other studies. Our multiple regression analysis showed that pain and fatigue were uniquely associated with the complaints of recalling and attentional deficits in patients with FM. On the other hand; age and sleep were uniquely associated with aspects of dyscognition involving delayed memory, construction, abstraction and arithmetic in older-aged women. Consistently wlth these findings, recently several writers have claimed that pain severity and pain-related fear and anxiety (pain catastrophising) exhibiting high somatic focus (somatisation) was associated with this disturbed attentional and recalling process in chronic pain patients and fibromyalgia $[29,30]$. And also it has been suggested that excessive fatigue associated with disturbed sleep might be another reason for the cognitive dysfunction observed in fibromyalgia patients [31]. Of interest in our study, sleep problems made a moderately significant contribution to the occurrence of cognitive complaints in patients with FM, this factor did not maintain a significant relation to cognitive function after the respective multiple regression analyses. This might be related to finding that, severity of sleep problems were significantly higher in our older-aged cohort than subjects with FM patients. As supported by the literature age-related increase in insomnia symptoms have been known problems in older aged people but further studies are needed to compare sleep disorders by objective measures between these groups $[32,33]$. Collectively all these findings showed us that STMS might be a useful and applicable test in clinical setting easily to distinguish subtypes of cognitive complaints in subjects with FM and in older-aged group and to identify their relationship with independent variables.

Also of note, our study failed to identify any association between cognitive complaints and depressive status. Many studies in the literature have reported that symptoms of depression made the strong contribution to complaints of cognitive deficits [33]. However, according to many studies this demonstrable cognitive dysfunction in patients with FM cannot be attributed solely to co-occurring psychiatric disorders such as depression, and that it is related with pain, fatigue and many to the impact that pain has on a patient [24]. According to study evaluating the psychometric properties and cut-off points of Beck Depression Inventory Scale for Turkish population, authors have determined the cut-off scores for the Turkish population revealing 0-12 for minimal, 13-18 for mild, 19-28 for moderate and 29-63 for severe depression [17]. And in our study we have found that in both groups total scores of BDI revealed mild depression. This might be due to the exclusion of the depressive patients at the beginning of our study. So; it was not surprising that all aspects of depression, as measured by the BDI, were not associated with reports of cognitive impairment. This has led us to think that some factors other than depression such as pain, fatigue and sleep were the major contributors of the cognitive dysfunction as supported by the literature [24].

The present study has some methodologic limitations. Because the experimental design is cross-sectional, the significant associations obtained do not provide information on causality so, further studies with larger samples of patients with long-term follow-up are required to confirm our results. And because the study relies on self-report measures, it is vulnerable to the effects of response style and impression management. However our results were similar in studies using objective testing methods. Considering the difficulty of these tests in clinical setting, STMS was easy to handle and might be useful screening test to identify differences between the groups and to put forward the relationship between cognitive complaints and clinical variables.

\section{Conclusion}

By means of covering a broad range of cognitive functions of STMS, mild cognitive impairment in patients with FMS and older-aged women could be demonstrated when compared to age-matched individuals in the general population. And the pattern of cognitive impairment also differed in subdimensions and underlying clinical factors. In other words we could obtain the similar results in other studies using objective neurophsychologic methods without using these tests that was the striking aspect of our study. Since it is important to characterize easily the nature of the dysfunction in clinical setting in order cope with this chronic disorder, our results might be useful in helping patients with FM and older-aged women. 
Citation: Öncü J, ilişer R, Kuran B (2015) Cognitive Complaints in Patients with Fibromyalgia Versus Older-Aged Women: Case Control Study. J Psychiatry 18: 242 doi: 10.4172/2378-5756.1000242

\section{Competing Interests}

The authors of this manuscript state that all of the authors have seen and agreed to the submitted version of the manuscript and with the listing of the authors. All authors have contributed substantially in the manuscript preparation, interpretation of results or study design and there weren't any potential conflicts of interest between the authors. The principal authors takes full responsibility for the data presented in this study, analysis of the data, conclusions, and conduct of the research, and had full access to those data and has maintained the right to publish any and all data independent of any third party.

\section{Authors' Contributions}

$\mathrm{JÖ}, \mathrm{RI}$, and BK participated in the design of this study. JÖ participated in the analysis and the interpretation of data and the writing of this manuscript and in the literature review and extraction. All of the authors read and approved the content of the manuscript.

\section{Acknowledgements}

This study was not supported by any funding or grant. The authors would like to thank all the patients participating in the study.

\section{Conflicts of Interest}

There weren't any potential conflicts of interest present.

\section{References}

1. Schmidt-Wilcke $T$, Wood $P$, Lürding $R$ (2010) Cognitive impairment in patients suffering from fibromyalgia. An underestimated problem. Schmerz 24: 46-53.

2. Tornero J, Vidal J (1999) Impacto social y económico de las enfermedades reumáticas: la discapacidad laboral. Rev Esp Reumatol 26: 347-366.

3. Buckhardt CS, Goldenberg D, Crofford L, Gerwin R, Gowens S, et al. (2005) Guideline for the management of fibromyalgia syndrome pain in adults and children. In Glenview (IL): American Pain Society (APS) 109. (Clinical practice guideline; no. 4).

4. Glass JM (2008) Fibromyalgia and cognition. J Clin Psychiatry 69: 2: 20-24.

5. Andreu J, Bosch R, Vázquez A, Masramon X, Rejas J, et al. (2009) Cognitive impairment in patients with Fibromyalgia syndrome as assessed by the MiniMental State Examination. BMC Musculoskeletal Disorders Dec 21: 10: 162.

6. Roth RS, Geisser ME, Goodvich MT, Dixon PJ (2005) Cognitive Complaints Are Associated With Depression, Fatigue, Female Sex, and Pain Catastrophizing in Patients With Chronic Pain. Arch Phys Med Rehabil 86: 1147-1154.

7. Park DC (2000) The basic mechanisms accounting for age-related decline in cognitive function. In Cognitive Aging: A Primer. Edited by Park DC, Schwarz N. Philadelphia: Psychology Press 3-22.

8. Grace GM, Nielson WR, Hopkins M, Berg MA (1999) Concentration and memory deficits in patients withfibromyalgia syndrome. J Clin Experimental Neuropsychol 21: 477-487.

9. Park DC, Smith AD, Lautenschlager G (1996) Mediators of long-term memory performance across the life span. Psychology Aging 11: 621-637.

10. Wolfe F (2010) New American College of Rheumatology Criteria for Fibromyalgia: A Twenty-Year Journey Arthritis Care \& Research 62: 583-584.

11. Wolfe F, Smythe HA, Yunus MB, Bennett RM, Bombardier C, et al. (1990) The American College of Rheumatology 1990 Criteria for the Classification of Fibromyalgia: Report of the Multicenter Criteria Committee. Arthritis Rheum 33: 160-172.

12. Rapp RSS, Legault C, Henderson VW, Brunner RL, Masaki K, et al. (2010) Subtypes of Mild Cognitive Impairment in Older Postmenopausal Women: The Women's Health Initiative Memory. Alzheimer Dis Assoc Disord 24: 248-255.

13. Kokmen E (1991) The Short Test of Mental Status. Arch Neurol 48: 725-728.

14. Tang-Wai DF, Knopman DS, Geda YE, Edland SD, Smith GE, et al. (2003) Comparison of the short test of mental status and the mini-mental state examination in mild cognitive impairment. Arch Neurol 60: 1777-81.

15. Carlsson MA (1983) Assessment of chronic pain. Aspects of the Reliability and Validity of the Visual Analogue Scale. Pain 16: 87-101.
16. Gencay-Can A, Can SS (2012) Validation of the Turkish version of the fatigue severity scale in patients with fibromyalgia. Rheumatol Int 32: 27-31.

17. Kapci EG, Uslu R, Turkcapar H, Karaoglan A (2008) Turkcapar Beck Depression Inventory II: evaluation of the psychometric properties and cut off points in a Turkish adult population. Depression and Anxiety 25: 104-110.

18. Stebbings S, Herbison P, Terrence CHD, Treharne GJ, Highton JA, et al. (2010) comparison of fatigue correlates in rheumatoid arthritis and osteoarthritis: disparity in associations with disability anxiety and sleep disturbance. Rheumatology 49: 361-367.

19. Kokmen E, Naessens JM, Offord KP (1987) A short test of mental status: description and preliminary results. Mayo Clinic Proceedings 62: 281-288.

20. Pericot-Nierga I, Hernández-Ferràndiz M, Lozano-Gallego M, Vilalta-Franch J Cruz-Reina Mdel M, et al. (2009) Cognitive profile in fibromyalgia. Comparison with a mild cognitive impairment group. Med Clin (Barc) 20: 133: 91-94.

21. Park DC (2000) The basic mechanisms accounting for age-related decline in cognitive function. In Cognitive Aging: A Primer. Edited by Park DC, Schwarz N Philadelphia: Psychology Press 3-22.

22. Glass JM, Park DC (2001) Cognitive dysfunction in fibromyalgia. Curr Rheumatol Rep 3: 123-127.

23. Crombez G, Eccleston C, Baeyens F, Eelen $P$ (1998) When somatic information threatens, catastrophic thinking enhances attentional interference. Pain 75 : 187-198.

24. Van Damme S, Crombez G, Eccleston C (2004) Disengagement from pain: the role of catastrophic thinking about pain. Pain 107: 70-76

25. Salthouse TA (1996) The processing-speed theory of adult age differences in cognition. Psychological Rev 103: 403-428.

26. Christensen $\mathrm{H}$ (2001) What cognitive changes can be expected with normal ageing? Australian and New Zealand Journal of Psychiatry 35: 768-775.

27. Goubert L, Crombez G, Van Damme S (2004) The role of neuroticism, pain catastrophizing and pain-related fear in vigilance to pain: a structural equations approach. Pain 107: 234-41.

28. Gracely RH, Geisser ME, Giesecke T (2004) Pain catastrophizing and neura responses to pain among persons with fibromyalgia. Brain 127: 1-9.

29. Cote KA, Moldofsky H (1997) Sleep, daytime symptoms, and cognitive performance in patients with fibromyalgia. J Rheumatol 24: 2014-2023.

30. Lindstrom V, Andersson K, Lintrup M, Holst G, Berglund J (2012) Prevalence of sleep problems and pain among the elderly in Sweden. J Nutr Health Aging 16: $180-183$

31. Salo P, Vahtera J, Ferrie JE, Akbaraly T, Goldberg M, et al. (2012) Trajectories of sleep complaints from early midlife to old age: longitudinal modeling study. Sleep. Nov 1: 35: 1559-1568.

32. Veiel HO (1997) A preliminary profile of neuropsychological deficits associated with major depressive disorder. J Clin Exp Neuropsychol 19: 587-603.

33. Williams RA, Hagerty BM, Cimprich B, Therrien B, Bay E, Oe H (2000) Changes in attention and short-term memory in depression. J Psychosom Res 34: $227-238$ 